\title{
Safety and efficacy of bleomycin/pingyangmycin-containing chemotherapy regimens for malignant germ cell tumor patients in the female genital system
}

\author{
Qianying Zhao' ${ }^{1}$, Dongyan $\mathrm{Cao}^{1}$, Mei Yu ${ }^{1}$, Jiaxin Yang ${ }^{1}$, Yongjian Liu ${ }^{2}$, Yang Xiang ${ }^{1}$, \\ Ming Wu ${ }^{1}$, Lingya Pan ${ }^{1}$, Jinghe Lang ${ }^{1}$, Kaifeng $X u^{2}$, Jiangna Han ${ }^{2}$ and Keng Shen ${ }^{1}$ \\ ${ }^{1}$ Department of Gynecology and Obstetrics, Peking Union Medical College Hospital, Chinese Academy of Medical Sciences \& \\ Peking Union Medical College, Beijing, China \\ 2 Department of Respiratory Medicine, Peking Union Medical College Hospital, Chinese Academy of Medical Sciences \& Peking \\ Union Medical College, Beijing, China \\ Correspondence to: Keng Shen, email: shenkeng@pumch.cn
}

Jiangna Han, email: 13520053104@139.com

Qianying Zhao, email: ofeizhaoqy@163.com

Keywords: bleomycin, medication safety, treatment efficacy, pulmonary toxicity, malignant germ cell tumor Received: October 15, $2016 \quad$ Accepted: January 09, $2017 \quad$ Published: February 02, 2017

\section{ABSTRACT}

Objectives: To comprehensively evaluate the safety and effectiveness of bleomycin/pingyangmycin-containing chemotherapy for female patients with malignant germ cell tumors in their genital system; to assess the diagnostic value of pulmonary function tests for bleomycin-induced pulmonary toxicity.

Methods: Data from a cohort of 120 patients, collected across 25 years, was reviewed. Chemotherapy-related adverse events were routinely monitored. Pulmonary toxicity was diagnosed and graded according to serial pulmonary function testing results, and potential impact factors were explored. Short-term remission probability and long-term prognosis were evaluated.

Results: Overall, $49.2 \%$ of the patients had pulmonary dysfunction, and the majority manifested as diffusion function impairment. A moderate reduction of carbon monoxide diffusion capacity was detected in $45.0 \%$ of all patients, and was severe in 3 patients. Thrombocytopenia, renal dysfunction, and accumulating dose of bleomycin/ pingyangmycin significantly increased the risk of lung injury $(P<0.05)$. Thorough surgical removal of tumors enhanced both remission and survival rate. Full-dose delivery of bleomycin/pingyangmycin and patients' sensitivity to chemotherapy also improved long-term survival $(P<0.05)$.

Conclusions: BPT could be sensitively detected and elaborately graded by PFTs, but the appropriate cut-off value for diagnosis needs further investigations. Timely recognition and control of renal dysfunction and thrombocytopenia could avail the patients of the opportunity to complete curative antineopalstic treatment. Prescriptive bleomycin/pingyangmycin-containing chemotherapy after optimal surgical resection could benefit MGCT patients maximally by improving both remission and survival rate.

\section{INTRODUCTION}

Malignant germ cell tumor (MGCT) is a rare entity in the female genital system and has a peak incidence in women under the age of 20 [1]. Due to cisplatin-based chemotherapy, the 5-year survival rate for patients with MGCTs exceeds $90 \%$ [2]. Given that $85 \%$ of metastatic GCTs are also cured and that the majority of patients are young adults, treatment-related complications become a core determinant for therapeutic plans [3].

Bleomycin is an attractive addition to combination chemotherapy regimens because of its broad activity and low myelotoxicity. The drug has been the mainstay for years in the treatment of MGCTs [4]. Meanwhile, bleomycin-induced pulmonary toxicity (BPT) has been commonly recognized since its clinical application from 
the early 1970s [5]. BPT may have immediate fatal effects or long-term clinical consequences for survivors with a long life expectancy [6].

However, consistent diagnostic criteria have yet to be developed. The incidence of BPT ranges from $2 \%$ to $46 \%$ due to diverse examination strategies that range from non-specific respiratory symptoms and less-sensitive radiographs to invasive lung biopsy. A number of potential risk factors for BPT have been revealed, including increasing age, underlying lung disease, smoking history, a cumulative bleomycin dose, renal insufficiency, radiation, supplemental oxygen exposure, and granulocyte colonystimulating factor (G-CSF) support $[3,6,7]$.

In addition, other therapeutic side effects (e.g., myelosuppression and renal dysfunction) might influence BPT severity as well as oncologists' clinical decisions. Therefore, we reviewed the records from a continuous cohort of female MGCTs in the genital system and prospectively followed these patients. The aim of this study was to assess both the safety and efficacy of PEB (cisplatin, etoposide, and bleomycin/pingyangmycin) or PVB (cisplatin, vincristine, and bleomycin/ pingyangmycin) chemotherapy with an emphasis on exploring the incidence and influential factors of BPT measured by serial pulmonary function test (PFT) results.

\section{PATIENTS AND METHODS}

The Patients' Record Database was searched for eligible patients between 1998 and 2014. Our final study group included all female patients with MGCTs in their genital system who were treated with a bleomycin/ pingyangmycin-containing regimen at our institution. Pingyangmycin, isolated from actinomycetes in Pingyang County (China), has a similar structure to the composition A5 of bleomycin compound and presents equal antineoplastic effect. MGCT diagnoses were confirmed by the pathological review of all specimens in our hospital, and all histological subtypes were eligible for this study.

Patients with FIGO (International Federation of Gynecology and Obstetrics) stage-I dysgerminoma and immature teratoma (grade 1) could be followed, together with those who did not receive a bleomycin/ pingyangmycin-containing regimen, were excluded. Standard front-line chemotherapy was either 3 or 4 cycles of PEB or PVB every 3 weeks (cisplatin $30-35 \mathrm{mg}$ / $\mathrm{m}^{2} / \mathrm{d}$ on days $1-3$, etoposide $100 \mathrm{mg} / \mathrm{m}^{2} / \mathrm{d}$ on days $1-3$, or vincristine $1.0-1.5 \mathrm{mg} / \mathrm{m}^{2} / \mathrm{d}$ on days $1-2$, bleomycin/ pingyangmycin $15 \mathrm{mg} / \mathrm{m}^{2} / \mathrm{d}$ on days $1-2$ or $20 \mathrm{mg} / \mathrm{m}^{2} / \mathrm{d}$ day $2 /$ weekly). The actual cycles that were applied might be flexible based on disease condition and therapeutic response. Usually, two courses were consolidated after serum tumor makers (e.g., CA125, AFP and HCG) declined to a normal level. Bleomycin/pingyangmycin was discontinued if (1) the patient's carbon monoxide diffusion capacity corrected for hemoglobin (DLCO) dropped below $70 \%$ of the predicted value; or (2) decreased $>20 \%$ from baseline level during treatment; and if (3) the lifetime dose limit of $250 \mathrm{mg} / \mathrm{m}^{2}$ was exceeded. A regimen with bleomycin/pingyangmycin omitted (e.g. cisplatin and etoposide, with or without vincristine) was subsequently substituted if necessary. The initial treatment was considered to be effective if the neoplasm was optimally excised and if the surveillance indicators (tumor markers, imaging and physical examinations) were all negative for at least 4 weeks after the completion of treatment. Side effects were under surveillance: complete blood count, liver and renal function, chest X-ray, PFT, glomerular filtration rate (GFR) or creatinine clearance rate $(\mathrm{CCr})$, and tumor markers were monitored. Patients who did not receive all their antineoplastic medications at our institution were omitted for inadequate records.

With PFTs we assessed the patients' forced vital capacity (FVC), forced expiratory volume in 1 second (FEV1), FEV1/FVC ratio, total lung capacity (TLC,) and DLCO. According to the Global Initiative for Chronic Obstructive Lung Disease (GOLD) criteria [8], obstructive pulmonary function impairment was defined as an FEV1/FVC ratio $<0.70$ and FEV $1<80 \%$ of the predicted value. Restrictive pulmonary function impairment and diffusion capacity impairment were defined according to the Common Terminology Criteria for Adverse Events (CTCAE) version 3.0 [9, 10]. Specifically, restrictive dysfunction was defined as TLC $<75 \%$ of the predicted value. Diffusion impairment was measured using the DLCO and the disease severity was graded from 0 to 5: (0) no impairment, DLCO $>90 \%$ of the predicted value; (1) mild impairment, $90 \% \geq$ DLCO $>75 \%$ of the predicted value; (2) moderate impairment, $75 \% \geq \mathrm{DLCO}>50 \%$ of the predicted value; (3) severe and undesirable impairment, $50 \% \geq \mathrm{DLCO}>25 \%$ of the predicted value; (4) life-threatening or disabling impairment, DLCO $<25 \%$ of predicted value; and (5) death. By definition, a pulmonary function impairment of grade 2 or higher $(\leq 75 \%$ ) can be considered abnormal and reflects physiological impairment [10]. Patients with underlying pulmonary dysfunction before chemotherapy were excluded.

Other treatment toxicities and potential risk factors for BPT were co-recorded, including age, prior lung disease, allergy history, renal function, cumulative bleomycin/pingyangmycin dose and delivery approach (intravenous, intramuscular or both), myelosuppression and the consequent use of G-CSF or blood transfusion and supplemental oxygen exposure. Tobacco and alcohol consumption information was not available for evaluation in our young female cohort. The lowest GFR or $\mathrm{CCr}$ was recorded and split by $80 \mathrm{ml} / \mathrm{min}$ for renal dysfunction diagnosis [3]. Aside from the total dose of bleomycin/pingyangmycin, the time when BPT initially occurred was also documented. Bone marrow toxicity was graded for each cycle according to CTCAE [9], 
Table 1: Clinical characteristics of enrolled patients

\begin{tabular}{|c|c|c|c|c|c|c|c|}
\hline \multirow[t]{2}{*}{ Clinical features } & \multicolumn{2}{|c|}{ All patients } & \multicolumn{2}{|c|}{ BPT patients } & \multicolumn{2}{|c|}{ Non-BPT patients } & \multirow[t]{2}{*}{$P$-value } \\
\hline & No. & $\%$ & No. & $\%$ & No. & $\%$ & \\
\hline Total & 120 & 100.0 & 59 & 100.0 & 61 & 100.0 & - \\
\hline \multicolumn{8}{|l|}{ Age, years } \\
\hline Median (Range) & \multicolumn{2}{|c|}{$20.7(6-45)$} & \multicolumn{2}{|c|}{$21.2(7-38)$} & \multicolumn{2}{|c|}{$20.3(6-45)$} & 0.984 \\
\hline Ethnic group & & & & & & & 1.000 \\
\hline Han & 111 & 92.5 & 55 & 93.2 & 56 & 91.8 & \\
\hline Minority & 9 & 7.5 & 4 & 6.8 & 5 & 8.2 & \\
\hline Prior lung disease & & & & & & & 1.000 \\
\hline Absent & 119 & 99.2 & 59 & 100.0 & 60 & 98.4 & \\
\hline Present & 1 & 0.8 & 0 & 0.0 & 1 & 1.6 & \\
\hline Allergic history & & & & & & & 0.645 \\
\hline Absent & 97 & 80.8 & 49 & 83.1 & 48 & 78.7 & \\
\hline Present & 23 & 19.2 & 10 & 16.9 & 13 & 21.3 & \\
\hline Histology & & & & & & & 0.302 \\
\hline Dysgerminoma & 31 & 25.8 & 12 & 20.3 & 19 & 31.1 & \\
\hline Non-dysgerminoma & 77 & 64.2 & 42 & 71.2 & 35 & 57.4 & \\
\hline Mixed MGCT & 12 & 10.0 & 5 & 8.5 & 7 & 11.5 & \\
\hline Stage & & & & & & & 1.000 \\
\hline FIGO I/II & 103 & 85.8 & 51 & 86.4 & 52 & 85.2 & \\
\hline FIGO III/IV & 17 & 14.2 & 8 & 13.6 & 9 & 14.8 & \\
\hline Regimen & & & & & & & 0.051 \\
\hline PEB & 100 & 83.3 & 48 & 81.3 & 52 & 85.3 & \\
\hline PVB & 12 & 10.0 & 4 & 6.8 & 8 & 13.1 & \\
\hline Both & 8 & 6.7 & 7 & 11.9 & 1 & 1.6 & \\
\hline Agent & & & & & & & 0.555 \\
\hline Bleomycin & 24 & 20.0 & 13 & 22.0 & 11 & 18.0 & \\
\hline Pingyangmycin & 90 & 75.0 & 42 & 71.2 & 48 & 78.7 & \\
\hline Both & 6 & 5.0 & 4 & 6.8 & 2 & 3.3 & \\
\hline Route & & & & & & & 0.026 \\
\hline Intravenous & 27 & 22.5 & 12 & 20.3 & 15 & 24.6 & \\
\hline Intramuscular & 83 & 69.2 & 38 & 64.4 & 45 & 73.8 & \\
\hline Both & 10 & 8.3 & 9 & 15.3 & 1 & 1.6 & \\
\hline Schedule & & & & & & & 0.018 \\
\hline Weekly & 60 & 50.0 & 24 & 40.7 & 36 & 59.0 & \\
\hline 21-day & 48 & 40.0 & 25 & 42.4 & 23 & 37.7 & \\
\hline Both & 12 & 10.0 & 10 & 16.9 & 2 & 3.3 & \\
\hline
\end{tabular}

Abbreviations: BPT, bleomycin induced pulmonary toxicity; FIGO, International Federation of Gynecology and Obstetrics; MGCT, malignant germ cell tumor; PEB/PVB, cisplatin, etoposide/ vincristine and bleomycin/ pingyangmycin

and the worst degree of each myelosuppression subtype was applied for assessment. G-CSF and component blood transfusion (erythrocytes or thrombocytes) was prescribed accordingly. History of major surgery following chemotherapy was used as a surrogate identifier of supplemental oxygen use, which had been previously cited as a risk factor for BPT $[3,11]$.

The majority of patients admitted in the last decade are regularly followed-up at our Outpatient Department or local referral hospitals, and long-term prognosis was analyzed in this cohort. The overall survival (OS) and disease-free survival (DFS) were estimated with an emphasis on the association with BPT. Age was dichotomized by the median age of the study cohort, and the total dosage was categorized based on whether the course number of the bleomycin/pingyangmycincontaining regimen exceeded the guideline recommendations.

Influential factors for BPT severity were evaluated univariately by log-rank tests or Fisher's exact tests with 95\% confidence interval (CI). Multivariate analysis was performed with the logistic regression method. Because 
of small event numbers, it was not possible to evaluate the effects of risk factors for obstructive and restrictive pulmonary function impairments. The observation time for OS ranged from the date of diagnosis to the date of death or the study end date, whichever occurred first. While the endpoint for DFS was either the date of first recurrence or the last follow-up starting from the completion of the first effective treatment. Potential clinical parameters for survival were analyzed univariately using the Kaplan-Meier method. A $P$-value $<0.05$ (twosided) was considered to be statistically significant. Statistical analyses were performed using Statistical Product and Service Solutions (SPSS) Statistics 20.0 (IBM Corporation, Armonk, New York, USA).

This study was approved by the institutional review board at Peking Union Medical College Hospital (PUMCH), Beijing, China, and the data of all patients were analyzed anonymously.

\section{RESULTS}

\section{Study population}

A review of the database revealed 308 female patients with MGCTs in their genital system who were treated at PUMCH during the specified study period. Eventually, 120 cases met the criteria of our study, and the selection process and reasons for the exclusion of patients are summarized in Figure 1.

\section{Clinical characteristics}

Table 1 shows the clinical features of the patients enrolled in the study, including four DSD (disorder of sex development) females (social gender). BPT incidence was similar in DSD and other patients $(P=1.000)$. The majority of the patients were Han Chinese who were diagnosed between age 10 and 30. Excluding 12 tumors with mix histologies, approximately $70 \%$ of the MGCTs

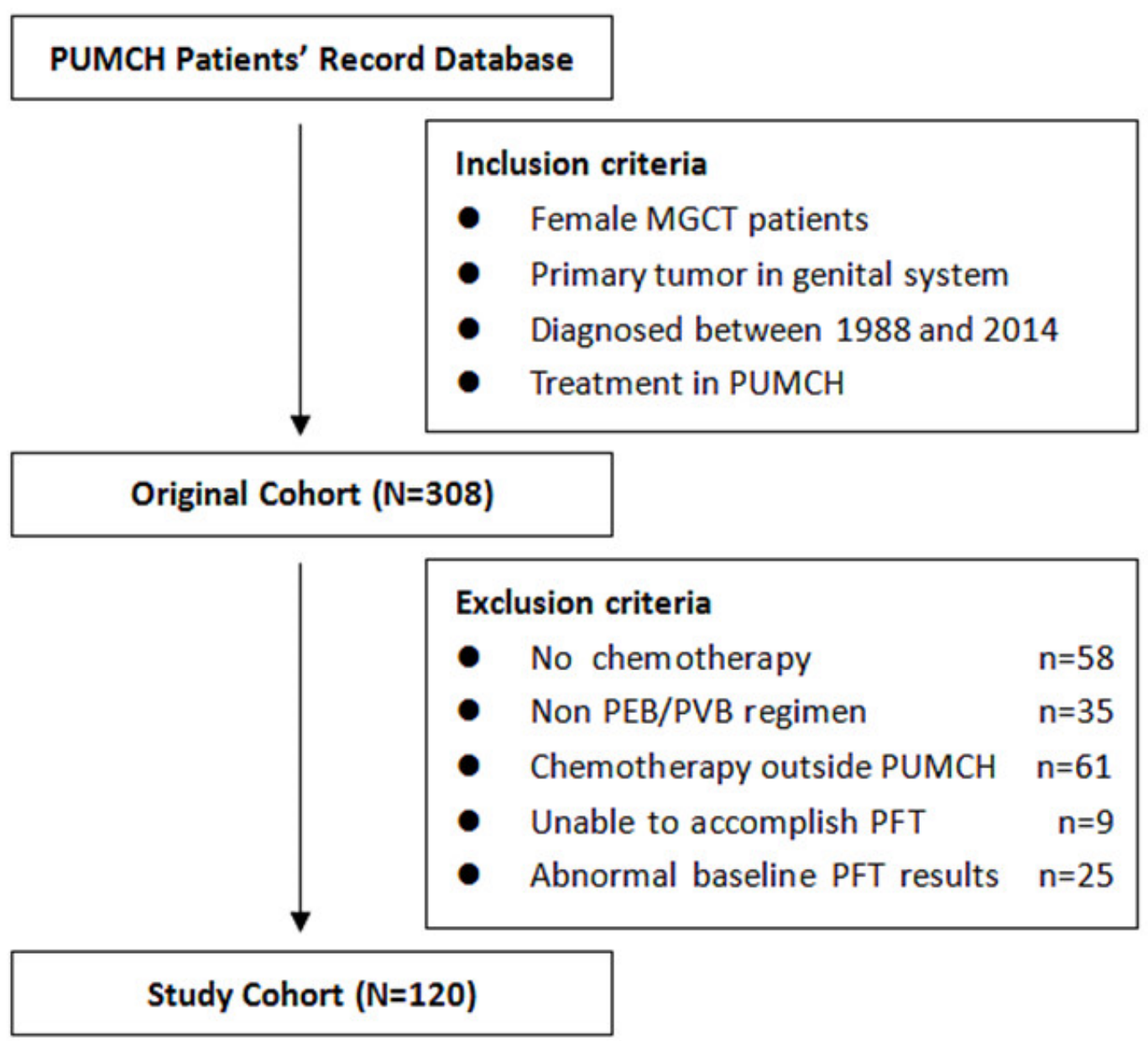

Figure 1: Process of patients' enrollment for this study. 
were non-dysgerminomas: embryonal carcinoma (1), endodermal sinus tumor (32), gonadoblastoma (1), and immature teratoma (43), with dysgerminomas in the remaining $30 \%$. Overall, $103(85.8 \%)$ patients merely had a neoplasm confined within the pelvis (i.e., FIGO stage I/ II). The majority $(83.3 \%)$ of patients only received PEB regimen, and domestic pingyangmycin was chose for most patients $(75.0 \%)$ because of economic reasons. Moreover, there were no significant differences found between the BPT and non-BPT group in terms of age, ethnic group, prior lung disease, allergic history, histology and stage $(P$ $>0.05)$.

\section{Bleomycin/pingyangmycin induced pulmonary toxicity}

Based on the worst PFT result of each patient from their first to the last administration of bleomycin/ pingyangmycin, only one case had mild and transient obstructive dysfunction, which was accompanied by diffusion impairment. Another 2 patients merely had TLC that dropped below $75 \%$ of the predicted value with normal FEV1, FEV1/FVC and DLCO. Diffusion impairment was present in 57 patients, and the severity was moderate (grade 2) in 54 patients and severe (grade 3 ) in 3 patients. None had a diffusion capacity impairment of grade 4 or 5 . In total, 59 patients $(49.2 \%)$ out of the 120 in the cohort had BPT defined as obstructive or restrictive dysfunction, or decreased DLCO (grade 2 or higher). Only three BPT (grade 2) patients had symptoms (e.g., cough, expectoration or thoracalgia), whereas two from the non-BPT group also complained of similar discomfort. Among the BPT group, 21 patients had an abnormal chest X-ray or computed tomography (CT) images, including two patients with symptoms. However, the manifestation was diverse and non-specific, such as nodules, bands, effusion or bulla. A typical image of BPT as fibrosis and organization was found in only one patient through high resolution CT. This patient had grade 2 DLCO reduction and cough. Furthermore, three patients had hypersensitive BPT (grade 2-3), which was characterized by a prompt DLCO reduction with less than $60 \mathrm{mg} / \mathrm{m}^{2}$ of bleomycin/pingyangmycin administration. Usually, DLCO would immediately drop more than $15 \%$ from the baseline, and their pulmonary function would not recover, even after drug withdrawal. Taking no account of this unique reaction type, the average cumulative doses of bleomycin/pingyangmycin in the BPT and non-BPT group were significantly different $(P<0.05)$. The average accumulated dosages for BPT patients were $200.4 \mathrm{mg} / \mathrm{m}^{2}$ and $120 \mathrm{mg} / \mathrm{m}^{2}$ through the intramuscular and intravenous administration route, respectively, and both dosages were significantly higher than those of the non-BPT group. In addition, PFTs from $55.9 \%$ of BPT patients would present abnormality right after the first course, whereas $54.2 \%$ of these patients had their worst pulmonary function after four or more courses of PEB/PVB (Figure 2). The incidence of BPT was similar between groups applied distinct route of bleomycin/pingyangmycin administration (i.e. intravenous and intravascular), or either chemotherapeutic schedule (i.e. 3-week and weekly). However, patients who had interchanged regimens would be more likely to develop BPT $(P<0.05$, Table 1$)$.

\section{Other common adverse events from treatment}

The lowest degrees of each bone marrow toxicity subtype for single patient were recorded and the pooled data were listed in Table 2. Myelosuppression in aspects of hemoglobin and thrombocyte was modest for the most part, whereas the worst granulopenia had reached a CTCAE grade 3 or 4 for $79.2 \%$ of patients. As a consequence, G-CSF was generally applied to patients with grade 3 or 4 granulopenia for therapeutic use, whereas prophylactic treatment was implemented for grade 4 cases. Patients with renal function impairment were $33.9 \%$ and $8.2 \%$ of the BPT and non-BPT groups, respectively. Abundant hydration was arranged due to cisplatin, and the
A

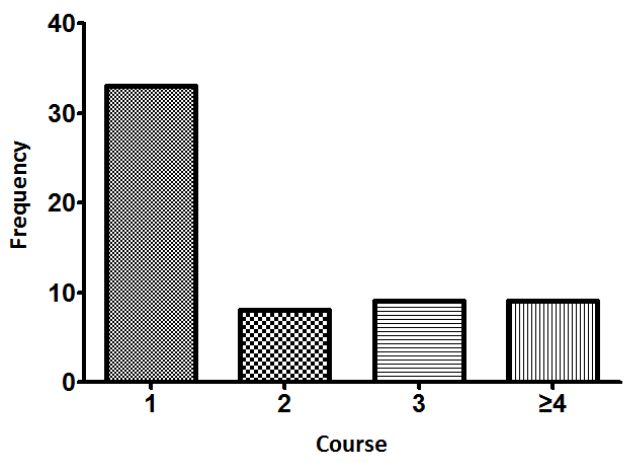

B

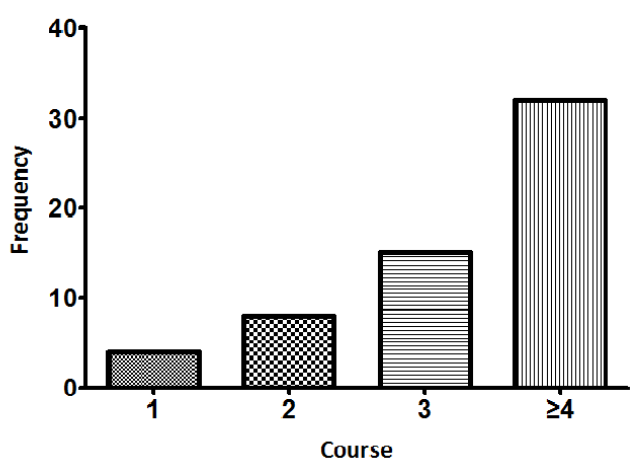

Figure 2: The occurrence time of A. the initial BPT; B. the most severe BPT. 
Table 2: Common chemotherapy-related adverse events

\begin{tabular}{|c|c|c|c|c|c|c|c|}
\hline & \multicolumn{2}{|c|}{ All Patients } & \multicolumn{2}{|c|}{ BPT Patients } & \multicolumn{2}{|c|}{ Non-BPT Patients } & \multirow[t]{2}{*}{$P$-value } \\
\hline & No. & $\%$ & No. & $\%$ & No. & $\%$ & \\
\hline Total & 120 & 100.0 & 59 & 100.0 & 61 & 100.0 & - \\
\hline \multicolumn{8}{|c|}{ Bone Marrow toxicity (CTCAE grade) } \\
\hline Hemoglobin & & & & & & & 0.000 \\
\hline$\leq 1$ & 77 & 64.2 & 33 & 55.9 & 44 & 72.1 & \\
\hline 2 & 22 & 18.3 & 11 & 18.6 & 11 & 18.0 & \\
\hline 3 & 13 & 10.8 & 8 & 13.6 & 5 & 8.3 & \\
\hline 4 & 8 & 6.7 & 7 & 11.9 & 1 & 1.6 & \\
\hline Leukocyte & & & & & & & 0.295 \\
\hline$\leq 1$ & 9 & 7.5 & 5 & 8.5 & 4 & 6.6 & \\
\hline 2 & 16 & 13.3 & 10 & 16.9 & 6 & 9.8 & \\
\hline 3 & 48 & 40.0 & 15 & 25.4 & 33 & 54.1 & \\
\hline 4 & 47 & 39.2 & 29 & 49.2 & 18 & 29.5 & \\
\hline Thrombocyte & & & & & & & 0.020 \\
\hline$\leq 1$ & 90 & 75.0 & 36 & 61.0 & 54 & 88.5 & \\
\hline 2 & 14 & 11.7 & 10 & 16.9 & 4 & 6.6 & \\
\hline 3 & 10 & 8.3 & 8 & 13.6 & 2 & 3.3 & \\
\hline 4 & 6 & 5.0 & 5 & 8.5 & 1 & 1.6 & \\
\hline \multicolumn{7}{|c|}{ Renal dysfunction (GFR or CCr) } & 0.001 \\
\hline$>80$ & 95 & 79.2 & 39 & 66.1 & 56 & 91.8 & \\
\hline$\leq 80$ & 25 & 20.8 & 20 & 33.9 & 5 & 8.2 & \\
\hline
\end{tabular}

Abbreviations: BPT, bleomycin/pingyangmycin induced pulmonary toxicity; CTCAE, common terminology criteria for adverse events; GFR, glomerular filtration rate; $\mathrm{CCr}$, creatinine clearance rate

Table 3: Univariate analysis of influential factors for BPT severity

\begin{tabular}{|l|c|l|c|}
\hline \multicolumn{1}{|c|}{ Impact factors } & $\boldsymbol{P}$-value & \multicolumn{1}{c|}{ Impact factors } & $\boldsymbol{P}$-value \\
\hline Epidemiologic information & & Chemotherapy detail & $\mathbf{0 . 0 4 3}$ \\
\hline Age at diagnosis & 0.335 & PEB/PVB & 0.755 \\
\hline Ethnic group & 0.856 & Bleomycin/pingyangmycin & $\mathbf{0 . 0 3 1}$ \\
\hline Individual history & & Weekly/monthly & $\mathbf{0 . 0 4 1}$ \\
\hline Prior lung disease & 1.000 & Route of administration & $\mathbf{0 . 0 0 0}$ \\
\hline Allergic history & 0.603 & Cumulative dose & 0.256 \\
\hline Disease state & & Average course interval & \\
\hline Stage of disease & 0.081 & Combined treatment & 0.331 \\
\hline Histological subtype & 0.741 & G-CSF & 0.904 \\
\hline Side effects of treatment & & Antibiotics & - \\
\hline Renal dysfunction & $\mathbf{0 . 0 0 1}$ & Blood transfusion & 0.116 \\
\hline Drug allergy & 0.332 & Erythrocyte & 0.035 \\
\hline Granulopenia & 0.176 & Thrombocyte & 0.067 \\
\hline Anemia & $\mathbf{0 . 0 4 1}$ & Chalybeate & 0.072 \\
\hline Thrombocytopenia & $\mathbf{0 . 0 0 0}$ & Post-chemotherapy surgery & \\
\hline
\end{tabular}

Abbreviations: BPT, bleomycin-induced pulmonary toxicity; PEB/PVB, cisplatin, etoposide/ vincristine and bleomycin; G-CSF, granulocyte colony stimulating factor

urine volume should be no less than $2500 \mathrm{ml}$ on the day cisplatin was administrated. Three patients suffered serious renal impairments (GFR $<60 \mathrm{ml} / \mathrm{min}$ ), and one of them had cisplatin dose induced, when the worst renal function was detected in the last chemotherapy cycle for the other two patients. Specifically, anemia and thrombocytopenia severity, together with renal insufficiency, significantly increased the risk of BPT by univariate evaluation $(P<$ $0.05)$. 
Table 4: Impact factors for remission and prognosis

\begin{tabular}{|l|c|c|c|}
\hline \multicolumn{1}{|c|}{ Impact factors } & \multicolumn{2}{c|}{ P-value } \\
\hline & Remission probability & Overall survival & Disease-free survival \\
\hline Age at diagnosis & 0.544 & 0.827 & 0.287 \\
\hline Ethnic group & 0.429 & 0.610 & 0.211 \\
\hline Stage & 0.258 & 0.247 & 0.384 \\
\hline Histological subtype & 0.721 & 0.318 & 0.711 \\
\hline Thoroughness of surgery & $\mathbf{0 . 0 3 9}$ & $\mathbf{0 . 0 0 9}$ & 0.093 \\
\hline PEB/PVB & 0.487 & 0.956 & 0.197 \\
\hline Bleomycin/pingyangmycin & $\mathbf{0 . 0 4 3}$ & 0.420 & 0.645 \\
\hline Route of administration & 0.649 & 0.744 & 0.660 \\
\hline Weekly/monthly & 0.334 & 0.238 & 0.242 \\
\hline Course interval & 0.369 & 0.911 & 0.393 \\
\hline Cumulative dose & 0.069 & $\mathbf{0 . 0 0 3}$ & 0.384 \\
\hline Renal dysfunction & $\mathbf{0 . 0 3 4}$ & 0.294 & 0.806 \\
\hline BPT severity & 0.370 & 0.214 & 0.464 \\
\hline Granulopenia & 0.343 & 0.610 & 0.127 \\
\hline Anemia & $\mathbf{0 . 0 0 3}$ & 0.130 & 0.339 \\
\hline Thrombocytopenia & 1.000 & 0.378 & - \\
\hline Remission & - & $\mathbf{0 . 0 0 0}$ & \\
\hline
\end{tabular}

Abbreviations: BPT, bleomycin-induced pulmonary toxicity; PEB/PVB, cisplatin, etoposide/ vincristine and bleomycin

\section{Impact factors for BPT severity}

BPT severity was graded based on the degree of DLCO decline. Risk factors were explored in regards to epidemiologic information, individual history, chemotherapy details, concomitant side effects and combined treatments. Table 3 depicts the results of univariate analyses for each potential hazard, excluding three hypersensitive BPT cases. The cumulative dose of bleomycin/pingyangmycin, the patients' renal function, hemoglobin and thrombocyte value, platelet transfusion, and chemotherapy regimen (PEB or PVB, weekly or monthly), and the administration route of bleomycin/ pingyangmycin were significantly associated with the degree of BPT $(p<0.05)$. However, multivariate analysis revealed that only increasing dosage, renal insufficiency and serious thrombocytopenia independently aggravated BPT after adjusting for other potential impact factors $(P$ $<0.05$ ).

Eighty percent of the patients with impaired renal eliminating ability had pathological DLCO reduction, whereas the proportion was merely $41.1 \%$ among patients with normal renal function. Moreover, all the three patients with severe BPT (grade 3) had a platelet level dropped below $75^{*} 10^{9} / \mathrm{L}$. Specifically, patients who had a grade 2 or higher degree of thrombocytopenia had an odds ratio (OR) of 4.93 for BPT occurrence compared to the combined normal and grade 1 group $(P<0.05)$.

\section{Remission probability and long-term prognosis}

With a satisfactory surgical resection of solid tumors and standard frontline chemotherapy, most patients (94.2\%) achieved remission immediately. However, the disease persisted for 7 remaining patients $(5.8 \%)$. Potential impact factors for remission probability detected univariately are listed in Table 4. However, multivariate analysis demonstrated that only surgery thoroughness was an independent predictor for short-term response $(P<0.05)$. Specifically, the remission probability for patients who had an optimal cytoreductive surgery was 9.9 times higher compared to patients whose tumors were not completely removed. In addition, BPT altered the oncologists' clinical decision: bleomycin/pingyangmycin dosage had been reduced in $42.3 \%$ among the BPT group $\left(20-60 \mathrm{mg} / \mathrm{m}^{2}\right.$ intramuscularly and $10-30 \mathrm{mg} / \mathrm{m}^{2}$ intravenously), whereas the remaining BPT patients had completed full-dose administration. More importantly, the latter group was more likely to remit with a curative remedy (OR 1.190, 95\% CI 1.003-1.413) without the exacerbation of BPT.

After a median observation time of 53.2 months (range, 2.1 to 139.7 months), two patients died due to ovarian cancer. The 5 -year OS rate was predicted to be $97.3 \%$ with an estimated median OS period of 11.2 years. Table 4 depicts the results of prognostic factors for OS investigated univariately. It indicated that optimal cytoreduction, sufficient delivery of bleomycin/ pingyangmycin and the initial response to therapy significantly improved survival $(P<0.05)$. Specifically, 
both deceased patients failed to achieve remission after the initial treatment. Until the end of the study, three patients had recurring diseases with a median relapse interval of 2.7 years. However, no prognostic factors that influenced recurrence were discovered, which probably occurred due to current limited events.

\section{DISCUSSION}

Diagnosed by PFTs, approximately half of the patients have developed pathological pulmonary dysfunction at our institution. In particular, diffusion capacity impairment was prevalent, whereas obstructive and restrictive function impairments were observed in only a few cases. BPT has been recognized ever since its early clinical trials, the diagnostic criteria have not yet been unified. Due to the diversity of examination strategies and enrolled populations, the incidence in prior studies ranged from $2 \%$ to $46 \%$ in various studies [12]. Compared to respiratory symptoms and chest radiographs, PFT showed higher sensitivity. However, some researchers blamed PFT for vast false positive results [13]. Though life-threatening BPT and cause-specific deaths were prevented from happening, the remission probability was decreased for our BPT patients who had a reduced-dose of bleomycin/pingyangmycin administration. We thus posited that grade 2 DLCO reduction ( $>25 \%$ ) would better be merely a warning sign, rather than an indicator for immediate drug cessation. On the other hand, a more precise grading of BPT severity might help determine a more appropriate cut-off value for clinical intervention. Specifically, consultation with specialists in respiratory medicine is valuable.

Other common chemotherapy-related complications were routinely monitored to maintain a safe level of medication. The most frequent and prominent myelosuppression was granulopenia, and G-CSF was prescribed when WBC dropped too much or too soon, or without timely recovery. Severe anemia and thrombocytopenia also deserve attention, and component blood transfusion should be supplemented accordingly. Renal function was seriously impaired in three patients, probably due to cisplatin. Urine volume is supposed to be monitored, and adequate hydration should be applied. Carboplatin may be a substitute when cisplatin had to be ceased.

In this study, we focused on the relationship between BPT and other side effects, which has seldom been assessed in other studies. To the best of our knowledge, hand-in-hand aggravation of thrombocytopenia and BPT was revealed for the first time. However, the mechanism underlying this interaction is unknown. Lung fibrosis and inflammatory reaction might lead to platelet consumption, or disordered coagulation and fibrinolysis may be important for the pathogenesis of pulmonary fibrosis $[14,15]$. We suggest timely management of severe thrombocytopenia, which might bring both circulatory and respiratory adverse events under better control. However, the definite clinical value of this relationship needs further investigation and validation. Between $50 \%$ and $70 \%$ of bleomycin is excreted unchanged by the kidneys. The half-life is $2-5 \mathrm{~h}$ in patients with normal renal function, which can increase to 30 hours in patients with reduced GFRs [16], and lead to longer lung exposure to the drug. In accordance with other studies [3, 12], our results demonstrated that decreased GFR or CCr was a predictor for BPT. Renal function can be compromised in a number of ways, especially by cisplatin, or by a secondary obstruction due to the abdominal tumor. In addition, our study confirmed there were dose-dependent effects for BPT severity detected by other studies $[3,12,17]$.

We also investigated other potential impact factors for BPT [2, 3, 6, 7, 12, 17-19], yet none of these factors were validated by our data. In particular, Gerson et al demonstrated that patients who received bleomycin in a continuous infusion did not have DLCO alterations, and other studies showed that continuous infusion might have less toxicity than bolus administration $[12,19]$. We failed to find a significant difference in BPT severity between single intravenous and intramuscular routes. However, lung injury might be worse when patients received both administration methods successively. Therefore, we suggest a consistent single delivery pattern of bleomycin/ pingyangmycin for each patient.

After all, the therapeutic efficacy is the primary concern, especially for a malignant disease with a good prognosis. Under standard guidelines, both the short-term remission and long-term survival rates were relatively high in our patients. Martin et al demonstrated that BPT resulted in a significant decrease in 5-year OS in patients with Hodgkin's lymphoma [6]. However, none of the treatment complications could explain the deaths in our study, which agreed with the results from Thomsen et al [2]. It is worth mentioning that the reduction of bleomycin/ pingyangmycin administration did have a reversed impact on remission probability in the BPT group, whereas the dosages within lifetime limit generally would not lead to lethal BPT. Moreover, sufficient delivery of bleomycin/ pingyangmycin improved OS. Collectively, a full-dose standard $\mathrm{PEB} / \mathrm{PVB}$ regimen is recommended, and a more practicable BPT diagnostic model should be established using prospective controlled diagnostic trials.

We acknowledge that our study has several limitations. For the sake of accuracy, a large number of transferred patients without systematic records of side reactions were excluded. The cohort effects might influence the estimates, and factors underlying nonprescriptive chemotherapy might affect the drug-induced toxicities. Secondly, even though BPT was monitored while bleomycin/pingyangmycin was given, the impact factors were simultaneously collected. Therefore, the causal relationship could hardly be confirmed 
in a chronological manner. In addition, long-term complications need more intensive observation.

In conclusion, BPT could be sensitively detected and elaborately graded by PFTs, but the testing specificity needs to be enhanced. Concomitant thrombocytopenia, renal dysfunction, and an excessive dose of bleomycin/ pingyangmycin significantly aggravate BPT. Therefore, simple and minimally invasive tests, such as a routine blood test, GFR and PFT should be monitored. With timely recognition and definitive control of treatment complications, patients would have more opportunities to be cured by integral PEB/PVB chemotherapy. Eventually, precision medicine could be achieved from a clinical perspective with treatment efficacy and safety comprehensively balanced for each patient.

\section{CONFLICTS OF INTEREST}

The authors declare no conflicts of interest.

\section{REFERENCES}

1. Arora RS, Alston RD, Eden TO, Geraci M, Birch JM. Comparative incidence patterns and trends of gonadal and extragonadal germ cell tumors in England, 1979 to 2003. Cancer. 2012;118:4290-7.

2. Thomsen FB, Bandak M, Thomsen MF, Lauritsen J, Christensen IJ, Daugaard G. Survival and toxicity in patients with disseminated germ cell cancer aged 40 years and older. Cancer. 2014;120:43-51.

3. O'Sullivan JM, Huddart RA, Norman AR, Nicholls J, Dearnaley DP, Horwich A. Predicting the risk of bleomycin lung toxicity in patients with germ-cell tumours. Ann Oncol. 2003;14:91-6.

4. Gatta G, Zigon G, Capocaccia R, Coebergh JW, Desandes E, Kaatsch P, Pastore G, Peris-Bonet R, Stiller CA; EUROCARE Working Group. Survival of European children and young adults with cancer diagnosed 19952002. Eur J Cancer. 2009;45:992-1005.

5. Liles A, Blatt J, Morris D, Wardrop R 3rd, Sharma A, Sznewajs A, Goldsby R; Children's Oncology Group. Monitoring pulmonary complications in long-term childhood cancer survivors: guidelines for the primary care physician. Cleve Clin J Med. 2008;75:531-9.

6. Martin WG, Ristow KM, Habermann TM, Colgan JP, Witzig TE, Ansell SM. Bleomycin pulmonary toxicity has a negative impact on the outcome of patients with Hodgkin's lymphoma. J Clin Oncol. 2005;23:7614-20.

7. Sleijfer S. Bleomycin-induced pneumonitis. Chest. 2001;120:617-24.
8. From the Global Strategy for the Diagnosis, Management and Prevention of COPD. Global Initiative for Chronic Obstructive Lung Disease (GOLD), 2008. http://www. goldcopd.org/.

9. National Cancer Institute. CTEP. CTCAE v3.0. NCI, 2009.

10. Mulder RL, Thönissen NM, van der Pal HJ, Bresser P, Hanselaar W, Koning CC, Oldenburger F, Heij HA, Caron $\mathrm{HN}$, Kremer LC. Pulmonary function impairment measured by pulmonary function tests in long-term survivors of childhood cancer. Thorax. 2011;66:1065-71.

11. Ingrassia TS 3rd, Ryu JH, Trastek VF, Rosenow EC 3rd. Oxygen-exacerbated bleomycin pulmonary toxicity. Mayo Clin Proc. 1991;66:173-8.

12. Azambuja E, Fleck JF, Batista RG, Menna Barreto SS. Bleomycin lung toxicity: who are the patients with increased risk? Pulm Pharmacol Ther. 2005;18(5):363-6.

13. Ng AK, Li S, Neuberg D, Chi R, Fisher DC, Silver B, Mauch PM. A prospective study of pulmonary function in Hodgkin's lymphoma patients. Ann Oncol. 2008;19:1754.

14. Noth I, Anstrom KJ, Calvert SB, de Andrade J, Flaherty KR, Glazer C, Kaner RJ, Olman MA; Idiopathic Pulmonary Fibrosis Clinical Research Network (IPFnet). A placebocontrolled randomized trial of warfarin in idiopathic pulmonary fibrosis. Am J Respir Crit Care Med. 2012; 186:88.

15. Kimura M, Tani K, Miyata J, Sato K, Hayashi A, Otsuka S, Urata $\mathrm{T}$, Sone S. The significance of cathepsins, thrombin and aminopeptidase in diffuse interstitial lung diseases. J Med Invest. 2005;52:93.

16. Alberts DS, Chen HS, Liu R, Himmelstein KJ, Mayersohn M, Perrier D, Gross J, Moon T, Broughton A, Salmon SE. Bleomycin pharmacokinetics in man. I. Intravenous administration. Cancer Chemother Pharmacol. 1978;1:17781.

17. Chaudhary UB, Haldas JR. Long-term complications of chemotherapy for germ cell tumours. Drugs. 2003;63:156577.

18. Saxman SB, Nichols CR, Einhorn LH. Pulmonary toxicity in patients with advanced-stage germ cell tumors receiving bleomycin with and without granulocyte colony stimulating factor. Chest. 1997;111:657-60.

19. Gerson R, Tellez Bernal E, Lazaro Leon M, Sanchez Forgach E, Garcia Irigoyen C, Gutierrez Godinez F, Garcia Gonzalez H. Low toxicity with continuous infusion of highdose bleomycin in poor prognostic testicular cancer. Am J Clin Oncol. 1993;16:323-6. 